\title{
Chemical Trends in Impurity Incorporation into $\mathrm{Si}(100)$
}

\author{
M. Ramamoorthy, E. L. Briggs, and J. Bernholc \\ Department of Physics, North Carolina State University, Raleigh, North Carolina 27695-8202
}

(Received 10 March 1998)

\begin{abstract}
Theoretical investigations of the adsorption, incorporation, and segregation of a number of common impurities at the $(2 \times 1)$-Si(100) surface reveal two qualitatively distinct classes of behavior. Some impurities prefer to adsorb in trenches between surface dimer rows. Their incorporation into the surface is highly unfavorable. Other impurities prefer to adsorb on top of surface dimer rows. Their incorporation into the surface is either energetically favorable or only marginally unfavorable. The results explain a number of experimental observations. [S0031-9007(98)06884-7]
\end{abstract}

PACS numbers: 61.72.-y

The $(2 \times 1)$ reconstructed $\mathrm{Si}(100)$ surface, which is preferred for growth of Si-based devices, has complex structural and electronic properties. It is covered by rows of dimerized threefold-coordinated $\mathrm{Si}$ atoms, with a high density of dangling bonds and anisotropic stress fields [1-3]. Correspondingly, the adsorption, incorporation, and segregation of impurities at this surface is very complex. Acceptors like Al, Ga, and In from group III are observed to prefer the adlayer [4,5], while donors like P and As from group $\mathrm{V}$ prefer to incorporate into the surface [6-8]. There are exceptions to these trends. In group III, B incorporates into the surface $[9,10]$ while, from group $\mathrm{V}, \mathrm{Sb}$ remains in the adlayer [4].

A number of investigations have addressed the incorporation of As from the adlayer into the $\mathrm{Si}(100)$ surface $[6,7]$. Two factors were found to influence the observed ordering [5,11] of group III impurities in the adlayer and the segregation $[12,13]$ of group $\mathrm{V}$ impurities to the surface: preference for threefold coordination and passivation of surface dangling bonds. In the case of carbon, strain effects $[14,15]$ arising from its large atomic-size mismatch with Si determine its segregation and ordering. However, the marked distinction between the behavior of group III and group V impurities at the $\mathrm{Si}(100)$ surface is not readily understood on the basis of the above considerations, as all of these impurities prefer threefold coordination and have atomic sizes covering a very large range. Furthermore, the mechanism of incorporation of other donors and acceptors into this surface remains to be addressed.

In this paper, we report quantitative calculations of the energetics of adsorption and incorporation of a number of acceptor, donor, and isovalent impurities at the $\mathrm{Si}(100)$ surface. $\mathrm{Al}, \mathrm{Ga}, \mathrm{In}$, and $\mathrm{Sn}$ exhibit qualitatively similar behavior. They prefer to adsorb in the trenches between surface dimer rows and their incorporation into the surface is highly unfavorable. Impurities in the other class (which include $\mathrm{C}, \mathrm{B}, \mathrm{P}, \mathrm{As}$, and $\mathrm{Sb}$ ) prefer to adsorb on top of surface dimer rows. Their incorporation into the surface is either energetically favorable or only marginally unfavorable. These results explain a number of experimental observations and provide information useful in atomistic modeling of the initial stages of growth and doping of submicron devices.

The total energies of the various structures were calculated using density functional theory [16], within the local density approximation. Norm-conserving pseudopotentials [17], real-space multigrid algorithms [18], and slab supercells with a $(4 \times 4)$ surface unit cell and $6-10 \mathrm{Si}$ layers were employed. The dangling bonds in the bottom surface layer were saturated by $\mathrm{H}$ atoms. Energy differences were found to be converged to within $0.05 \mathrm{eV}$ with a real space grid spacing of $0.6(0.4)$ a.u. for group V (III) impurities, a vacuum layer thickness of 13.5 a.u., six $\mathrm{Si}$ layers, and one $k$ point at $\Gamma$. Our results for energy differences between different addimer configurations for $\mathrm{Si}$ and $\mathrm{Al}$ on $\mathrm{Si}(100)$ are in excellent agreement with prior work [11,19]. A conservative estimate of our error bars is $0.1 \mathrm{eV}$.

At the clean $\mathrm{Si}(100)$ surface, the surface $\mathrm{Si}$ atoms order in rows of dimers and are only threefold coordinated [2]. The energy of this surface is further reduced by buckling of the dimers [1], which opens up a Peierls' gap in this initially metallic system. In each dimer one atom is raised and accepts charge from the other atom that, in turn, is lowered from its initially symmetric position. The relaxed geometry we obtained for the clean $\mathrm{Si}(100)$ surface showed the expected $c(4 \times 2)$ ordering of buckled Si dimers [1].

We now compare the adsorption of $\mathrm{Al}, \mathrm{Ga}$, and $\mathrm{In}$, which are acceptors, to that of $\mathrm{P}, \mathrm{As}$, and $\mathrm{Sb}$ of group $\mathrm{V}$, which are donors. All of these impurities adsorb as dimers, with each impurity having threefold coordination and saturating two surface dangling bonds. Four stable addimer configurations are known on $\mathrm{Si}(100)$, with positions either on top of dimer rows or in the trenches, and oriented either parallel or perpendicular to the underlying Si dimers [19]. The relative energetics of these configurations separates these impurities into two distinct classes. Group III addimers prefer to adsorb in a trench, oriented parallel to the surface Si dimers [see Fig. 1(a)]. Displacing the addimer to the top of a dimer row, while maintaining its orientation, costs about $0.3-0.5 \mathrm{eV}$. Rotating the addimer to a perpendicular orientation, whether in a trench or on top of a row, costs at least $1.0 \mathrm{eV}$. Group V addimers, on the other hand, 
(a)
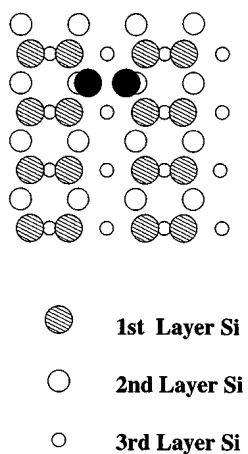

FIG. 1. The two generic equilibrium modes of adsorption of donor and acceptor dimers on $\operatorname{Si}(100)$.

prefer the configurations on top of the dimer rows over those in the trenches. Their equilibrium orientation is perpendicular to the surface Si dimers, as shown schematically in Fig. 1(b). Rotating the addimer to a parallel configuration costs about $0.5 \mathrm{eV}$, while both configurations in the trenches are more than $1.0 \mathrm{eV}$ higher in energy.

Next, the energy of incorporation of each impurity addimer $\left(X_{2}\right)$ into the near-surface region was calculated. Two qualitatively different processes were investigated, where the impurity addimer incorporates into the surface layer as (i) one unit, ejecting a surface Si dimer which moves into the adlayer, or (ii) two substitutional isolated impurities in the surface layer or in a near-surface layer, resulting in the ejection of two host $\mathrm{Si}$ atoms, which move into the adlayer. In terms of the supercell energies, the energy change for the process (i) is given by

$$
\Delta E=E_{S}\left[\left(X_{2}\right)^{\mathrm{inc}}\right]+E_{S}\left[\left(\mathrm{Si}_{2}\right)^{\mathrm{ad}}\right]-E_{S}\left[\left(X_{2}\right)^{\mathrm{ad}}\right]-E_{S}
$$

and that for process (ii) is given by

$\Delta E=2 E_{S}\left[(X)^{\mathrm{inc}}\right]+E_{S}\left[\left(\mathrm{Si}_{2}\right)^{\mathrm{ad}}\right]-E_{S}\left[\left(X_{2}\right)^{\mathrm{ad}}\right]-2 E_{S}$.

Here, $E_{S}$ stands for the energy of the clean surface supercell. Impurity configurations near the surface are indicated in parentheses, and "ad" and "inc" indicate adsorbed and incorporated configurations, respectively. The $\mathrm{Si}$ addimer prefers to adsorb on top of a dimer row with the orientations parallel or perpendicular to the surface Si dimers being within $0.1 \mathrm{eV}$ of each other [19]. With the above convention, the energy of its exchange with a surface Si dimer is zero. Process (i) has already been observed experimentally [6,8].

The calculated incorporation energies reveal striking trends. The six impurities ( $\mathrm{P}, \mathrm{As}, \mathrm{Sb}, \mathrm{Al}, \mathrm{Ga}$, and $\mathrm{In}$ ) discussed thus far are all well-known surfactants [20], due to their preference for threefold coordination. Their most favorable configurations are either in the adlayer or in the top surface layer. In each case, subsurface incorporation is higher in energy by at least $1.0 \mathrm{eV}$ per impurity pair. The energies of incorporation from the adlayer into the surface layer, on the other hand, fall into two distinct classes. They are greater by $0.5-1.0 \mathrm{eV}$ for group III impurities over those of group $\mathrm{V}$ impurities.

The results for the first mode of incorporation (i), where the impurity addimer replaces a surface Si dimer, are plotted in Fig. 2(a). In this mode, there is relatively little change in the chemical bonding configuration of the impurity dimer. For $\mathrm{P}$ and As, the process is favorable by about $0.2 \mathrm{eV}$ per addimer, while for $\mathrm{Sb}$ it is unfavorable by about $0.1 \mathrm{eV}$ per addimer. However, for group III impurities this process is unfavorable by $0.5-1.3 \mathrm{eV}$. In Fig. 2(b) the results for the second mode of incorporation (ii) are given. Here, two impurities replace $\mathrm{Si}$ atoms in two distinct Si dimers in the surface layer, giving rise to two impurity-Si heterodimers. In this mode, $\mathrm{Al}$ and $\mathrm{Ga}$ lose more than $0.5 \mathrm{eV}$ per addimer, while $\mathrm{P}$ and As gain about $0.5 \mathrm{eV}$ per addimer. Even the atoms that have big atomic size mismatches with $\mathrm{Si}$ preserve this trend; $\mathrm{Sb}$ gains only about $0.1 \mathrm{eV}$, while In loses almost $1.0 \mathrm{eV}$.

We now turn to boron. The equilibrium configuration for the $\mathrm{B}$ addimer is very similar to that of $\mathrm{P}, \mathrm{As}$, and $\mathrm{Sb}$.
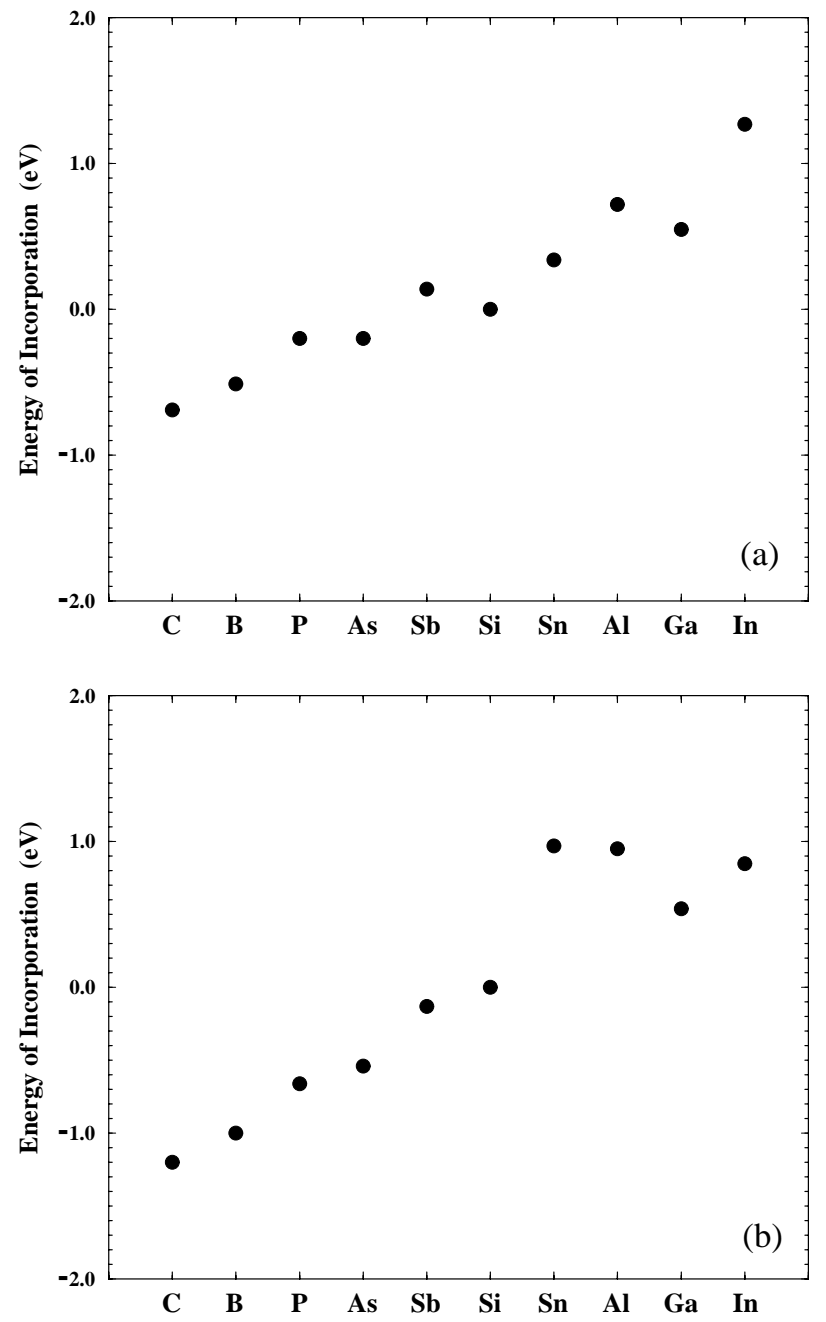

FIG. 2. (a) Energy of incorporation of each element as one surface impurity dimer on $\mathrm{Si}(100)$; (b) Energy of incorporation of each element as two surface impurity-Si heterodimers on $\operatorname{Si}(100)$. 
Further, like $\mathrm{P}$ and $\mathrm{As}$, a $\mathrm{B}$ addimer prefers to incorporate into the surface. The energy gain for the formation of two isolated surface B-Si heterodimers is $1.0 \mathrm{eV}$, significantly more than the $0.5 \mathrm{eV}$ gained on the formation of a surface B dimer. However, boron is anomalous compared with $\mathrm{P}$ and $\mathrm{As}$ as it prefers to incorporate into the second layer as well. The energy of the isolated second-layer impurity is almost degenerate with that in the first layer. Two second-layer impurities bind by about $0.2 \mathrm{eV}$ when they form a pair. This pair has two nearly degenerate orientations, parallel or perpendicular to the surface $\mathrm{Si}$ dimers, as shown in Fig. 3. Incorporation into the third layer or any deeper layer is unfavorable by over $1.0 \mathrm{eV}$ per pair, in common with the other impurities considered.

It is thus clear that the adsorption and incorporation of the above donors and acceptors at $\mathrm{Si}(100)$ fall into two distinct classes. To explore the broader relevance of this classification, we investigated $\mathrm{C}$ and $\mathrm{Sn}$, which are isoelectronic with $\mathrm{Si}$. Carbon is found to behave like $\mathrm{P}$, As, and B. It prefers adsorption on top of the dimer rows as opposed to the trenches, and is favored to incorporate into the surface. Two isolated $\mathrm{Si}-\mathrm{C}$ heterodimers are more stable than a surface $\mathrm{C}-\mathrm{C}$ dimer. However, like B, C favors both surface and subsurface incorporation. Incorporation of a $\mathrm{C}$ addimer into the first, second, or third layer results in a gain of $1.0 \mathrm{eV}$. On the other hand, the $\mathrm{Sn}$ dimer prefers to adsorb in the trenches between dimer rows, oriented like $\mathrm{Al}, \mathrm{Ga}$, and In. Furthermore, its incorporation into the surface is unfavorable by over $0.3 \mathrm{eV}$ per pair and subsurface incorporation is even more unfavorable.

In all adlayer and surface configurations above, the impurities are threefold coordinated and have atomic sizes covering a wide range. Therefore, coordination or strain effects do not explain the results. Valence is not the distinguishing feature either, as $\mathrm{B}$ and $\mathrm{C}$ fall into the same class as $\mathrm{P}, \mathrm{As}$, and $\mathrm{Sb}$, while $\mathrm{Sn}$ falls into the same class as $\mathrm{Al}, \mathrm{Ga}$, and In. Nor is there a correlation of the results with addimer bond lengths. For instance, in equilibrium, (a)

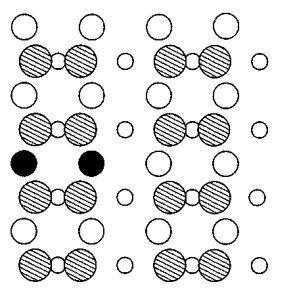

(b)

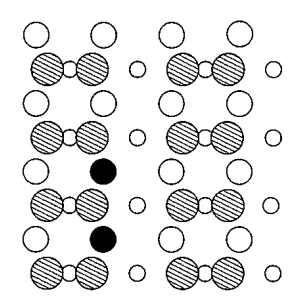

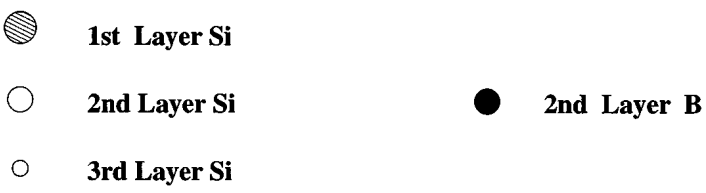

FIG. 3. The two nearly degenerate lowest energy modes of incorporation of B atoms, which form pairs in the second layer, underneath a row of surface Si dimers. the $\mathrm{Sb}$-Sb addimer's bond length differs from that of the In-In addimer by only about $0.1 \AA$ and yet their configurations are distinct.

The quantitative results on the interaction of the impurities with $\mathrm{Si}(100)$, which were obtained using fully quantum mechanical calculations, are now correlated with empirical knowledge regarding their chemical properties. If the admixture of an impurity $X$ from the adlayer into the surface layer in isolated substitutional configurations is energetically favorable (unfavorable), it indicates that two surface $\mathrm{Si}-X$ bonds are stronger (weaker) than a $X-X$ bond and a $\mathrm{Si}-\mathrm{Si}$ bond. In the binary phase diagrams [21] of each of the above impurities with $\mathrm{Si}$, it is seen that $\mathrm{P}, \mathrm{As}, \mathrm{Sb}, \mathrm{B}$, and $\mathrm{C}$ do react exothermically with bulk $\mathrm{Si}$ and form a number of stoichiometric compounds, while $\mathrm{Al}, \mathrm{Ga}, \mathrm{In}$, and Sn have extremely small miscibility with $\mathrm{Si}$ at any temperature. Thus the results that we obtained are consistent with bulk data that indicate significant differences in the strengths of the relevant chemical bonds.

A number of factors influence the strength of a heteropolar bond between two elements, one of which is the extent of charge transfer when the bond is formed. Generally, this is quantified using electronegativity tables. In Pauling's scale, $\mathrm{Si}$ is assigned an electronegativity that is higher than those corresponding to $\mathrm{Al}, \mathrm{Ga}$, and $\mathrm{In}$, but lower than those of C, B, P, As, Sb, and Sn. Again, this empirical grouping exhibits rough correlation with our results. When the relative energies of different surface configurations of these impurities are examined, subtle variations related to varying charge-transfer effects are noted. A surface impurity-Si heterodimer may be flat or it may be buckled, with the top atom accepting charge from the bottom atom [1]. For P, As, and Sb, the stable heterodimer configurations are all buckled, and the impurity occupies the top position in the lowest energy configuration. The other buckled configuration is about $0.3 \mathrm{eV}$ higher in energy. However, the buckled configuration with the impurity atom in the top position is unstable for $\mathrm{Al}, \mathrm{Ga}$, and In. The equilibrium configuration is nearly flat and favored by about $0.3 \mathrm{eV}$ over that with the impurity in the bottom position. The small atomic size of $\mathrm{B}$ and $\mathrm{C}$ relative to $\mathrm{Si}$ results in only the flat configuration of the corresponding heterodimer being stable.

Our results explain a variety of experimental investigations. Indeed $\mathrm{Al}, \mathrm{Ga}$, and In of group III prefer the adlayer. In group $\mathrm{V}, \mathrm{P}$ and As readily incorporate into the surface $[6,8]$, while $\mathrm{Sb}$ does not incorporate to any significant extent [4]. For B, different models of subsurface $\mathrm{B}$ incorporation into the second [9] or the third layer [10] of the surface have been proposed in the literature. Our calculations support the former model over the latter. The results for $\mathrm{C}$ and $\mathrm{Sn}$ are also consistent with published theory and experiment $[4,14,15]$.

One intriguing result of the calculations is that $\mathrm{P}-\mathrm{Si}$ and As-Si heterodimers in the surface are more stable than P-P and As-As dimers, respectively. Generally, As incorporation has been investigated [6] at moderate 
temperatures $\left(\sim 400-500{ }^{\circ} \mathrm{C}\right)$ via deposition of $\mathrm{As}_{2}$ and $\mathrm{As}_{4}$ molecules and only As dimer incorporation was observed. However, in a recent investigation [8] of $\mathrm{P}$ incorporation via decomposition of $\mathrm{PH}_{3}$ at about $650{ }^{\circ} \mathrm{C}$, extensive coverage of the surface by both P-Si and P-P structures was observed. It is important to understand the nature of the kinetic effects that favor incorporation of the impurities as dimers or as heterodimers.

Native point defects assist impurity incorporation, much as they assist impurity migration in bulk Si [22]. We report here the results of our systematic investigations of the formation energies of native point defects at and near the surface. The lowest energy defects were Si addimers and surface divacancies, with formation energies of only 0.1 and $0.2 \mathrm{eV}$, respectively. Si adatoms require $0.5 \mathrm{eV}$ to form, and vacancies in both the first and second layers require about $0.9 \mathrm{eV}$. Vacancies and interstitials that are deeper than the second surface layer have formation energies greater than $1.5 \mathrm{eV}$. These values are lower than those of bulk vacancies and interstitials, which are 3.3 and $3.9 \mathrm{eV}$, respectively.

The low formation energies of surface divacancies make them prevalent at temperatures as low as $300 \mathrm{~K}[23,24]$. Furthermore, extensive migration of impurity addimers has been observed at $450 \mathrm{~K}$ [25], implying a low diffusion activation energy barrier. A migrating $\mathrm{P}$ or As addimer can thus enter the surface as one unit, replacing a surface divacancy. If the impurities are deposited as molecules, whether elemental or as compounds, the formation of a Siimpurity heterodimer would first require the decomposition of the appropriate molecule or an "atomic exchange" process. In the decomposition case, each impurity atom that is released would need to replace a surface vacancy or kick out a surface $\mathrm{Si}$ atom, forming a $\mathrm{Si}$ adatom in turn. The formation energies of two $\mathrm{Si}$ adatoms is over $1.0 \mathrm{eV}$ and that of two near-surface vacancies is about $1.8 \mathrm{eV}$. Therefore, it is clear that the decomposition pathway is energetically unfavorable. Turning to an atomic exchange process, it would be competitive only if a low energy pathway that minimized the number of bonds existed for the case of the adsorbed dimers. The experimental data suggest that a low energy pathway does not exist.

Our results for the adsorption of group III impurities are in excellent agreement with earlier calculations (Refs. [5] and [11]). We are also in qualitative accord with $\mathrm{Yu}$ et al. (Ref. [7]) for the adsorption and incorporation of As, but there are significant quantitative differences as they used smaller $(2 \times 2)$ supercells. To the best of our knowledge, ours is the first comprehensive theoretical investigation of impurity incorporation into $\mathrm{Si}(100)$.

In conclusion, we have found that the adsorption and incorporation of a number of common impurities in Si fall into two distinct equivalence classes. Within each group, atomic-size differences result in quantitative shifts of the incorporation energies. The majority of these impurities, which include donors, acceptors, and isoelectronic impurities, are surfactants, in that they prefer the adlayer or surface layer. Boron and carbon alone, which are first-row elements, prefer subsurface incorporation. Kinetic effects play an important role in determining the distribution of incorporated configurations. Our results provide a systematic explanation of a number of experimental observations.

[1] D. J. Chadi, Phys. Rev. Lett. 43, 43 (1979).

[2] R. M. Tromp, R. J. Hamers, and J. E. Demuth, Phys. Rev. Lett. 55, 1303 (1985).

[3] O. L. Alerhand et al., Phys. Rev. Lett. 61, 1973 (1988).

[4] J. Nogami, A. A. Baski, and C.F. Quate, J. Vac. Sci. Technol. A 8, 245 (1990); Appl. Phys. Lett. 53, 2086 (1991); 58, 475 (1991); A. A. Baski, J. Nogami, and C. F. Quate, Phys. Rev. B 43, 9316 (1991); 44, 1415 (1991); A. A. Baski, C. F. Quate, and J. Nogami, Phys. Rev. B 44, 11167 (1991).

[5] J. E. Northrup et al., Phys. Rev. B 44, 13799 (1991).

[6] R. D. Bringans, D. K. Biegelsen, and L.E. Swartz, Phys. Rev. B 44, 3054 (1991).

[7] B. Yu and A. Oshiyama, Phys. Rev. Lett. 71, 585 (1993), and references therein.

[8] Y. Wang, X. Chen, and R. J. Hamers, Phys. Rev. B 50, 4534 (1994).

[9] Y. Wang, R. J. Hamers, and E. Kaxiras, Phys. Rev. Lett. 74, 403 (1995).

[10] Z. Zhang et al., J. Vac. Sci. Technol. B 14, 2684 (1996).

[11] G. Brocks, P. J. Kelly, and R. Car, Phys. Rev. Lett. 70, 2786 (1993); J. Vac. Sci. Technol. B 12, 2705 (1994).

[12] R. I. G. Uhrberg et al., Phys. Rev. Lett. 56, 520 (1986).

[13] R. D. Bringans, Crit. Rev. Solid State Mater. Sci. 17, 353 (1992).

[14] J. Tersoff, Phys. Rev. Lett. 74, 5080 (1995).

[15] P.C. Kelires and E. Kaxiras, Phys. Rev. Lett. 78, 3479 (1997), and references therein.

[16] W. Kohn and L. J. Sham, Phys. Rev. 140, A1133 (1965).

[17] D. R. Hamann, M. Schluter, and C. Chiang, Phys. Rev. Lett. 43, 1494 (1979).

[18] E. L. Briggs, D. J. Sullivan, and J. Bernholc, Phys. Rev. B 52, R5471 (1995); Phys. Rev. B 54, 14362 (1996).

[19] G. Brocks and P. J. Kelly, Phys. Rev. Lett. 76, 2362 (1996).

[20] M. Copel et al., Phys. Rev. Lett. 63, 632 (1989).

[21] A. S. Berezhnoi, Silicon and its Binary Systems (Consultants Bureau, New York, 1960).

[22] P. M. Fahey, P. B. Griffith, and J. D. Plummer, Rev. Mod. Phys. 61, 289 (1989), and references therein.

[23] Y. W. Mo et al., Phys. Rev. Lett. 66, 1998 (1991).

[24] J. Wang, T. A. Arias, and J. D. Joannopoulos, Phys. Rev. B 47, 10497 (1993).

[25] Y. W. Mo, Phys. Rev. Lett. 71, 2923 (1993). 